\title{
Grafting voltage and pharmacological sensitivity in potassium channels
}

\author{
Xi Lan ${ }^{1,3, *}$, Chunyan $\mathrm{Fan}^{2,3, *}$, Wei $\mathrm{Ji}^{2,3}$, Fuyun Tian ${ }^{1,3}$, Tao $\mathrm{Xu}^{2,3}$, Zhaobing Gao ${ }^{1,3}$ \\ ${ }^{I}$ CAS Key Laboratory of Receptor Research, Shanghai Institute of Materia Medica, Chinese Academy of Sciences, 555 Zuchongzhi \\ Road, Shanghai 201203, China; ${ }^{2}$ National Key Laboratory of Biomacromolecules, Institute of Biophysics, Chinese Academy of Sciences, \\ 5 Datun Road, Beijing 100101, China; ${ }^{3}$ University of Chinese Academy of Sciences, 19 A Yuquan Road, Beijing 100049, China
}

A classical voltage-gated ion channel consists of four voltage-sensing domains (VSDs). However, the roles of each VSD in the channels remain elusive. We developed a GVTDT (Graft VSD To Dimeric TASK3 channels that lack endogenous VSDs) strategy to produce voltage-gated channels with a reduced number of VSDs. TASK3 channels exhibit a high host tolerance to VSDs of various voltage-gated ion channels without interfering with the intrinsic properties of the TASK3 selectivity filter. The constructed channels, exemplified by the channels grafted with one or two VSDs from Kv7.1 channels, exhibit classical voltage sensitivity, including voltage-dependent opening and closing. Furthermore, the grafted Kv7.1 VSD transfers the potentiation activity of benzbromarone, an activator that acts on the VSDs of the donor channels, to the constructed channels. Our study indicates that one VSD is sufficient to voltage-dependently gate the pore and provides new insight into the roles of VSDs.

Keywords: voltage-gated channels; K2P channels; voltage-sensing domain; graft

Cell Research (2016) 26:935-945. doi:10.1038/cr.2016.57; published online 13 May 2016

\section{Introduction}

A classical voltage-gated potassium (Kv) channel consists of four voltage-sensing domains (VSDs) that perceive changes in the membrane potential and open or close the channels [1-3]. The VSD is formed by four transmembrane segments (S1-S4), and S4 contains 4-8 positively charged residues [4-7]. However, different from $\mathrm{Kv}$ channels, a voltage-gated proton channel Hv1 consists of two VSDs, and a voltage sensor-containing phosphatase Ci-VSP contains one VSD, suggesting that a single VSD can sense changes in the membrane potential and function independently [8-10]. Discoveries of voltage-sensing proteins with less than four VSDs raised a fundamental question: how many VSDs are necessary to

\footnotetext{
*These two authors contributed equally to this work. Correspondence: Zhaobing $\mathrm{Gao}^{\mathrm{a}}$, Tao $\mathrm{Xu}^{\mathrm{b}}$

${ }^{\mathrm{a}}$ Tel: +86-21-20239067; Fax: +86-21-20239067

E-mail: zbgao@simm.ac.cn

${ }^{\mathrm{b}} \mathrm{Tel}$ : +86-10-64889872

E-mail: xutao@ibp.ac.cn

Received 24 November 2015; revised 4 March 2016; accepted 29 March 2016; published online 13 May 2016
}

confer the voltage sensitivity in one Kv channel [11]?

In a model proposed by Hodgkin and Huxley [12], all four VSDs must be in an activated position to open one voltage-gated channel, whereas the downward motion of only one VSD is sufficient to close the channel. Supportively, the study in concatenated heterotetramers of Shaker channels with stoichiometry of 1 wild-type: 3 neutral S4 segments suggests that when three of VSDs are in the activated position, the fourth VSD is capable of gating the channel by itself $[13,14]$. KvLm is a prokaryotic Kv channel from Listeria monocytogenes, characterized by high structural independence of the VSDs and the pore domain [15]. Taking advantage of this unique property, functional heteromers of the KvLm pore domain, assembled with one, two, or three VSDs, were produced by in vitro transcription and translation [16]. The results from this study suggested that three VSDs are sufficient to gate the channel, but all four sensors are required to close the channel [16]. The work on KvLm channels shed new light on the roles of VSD. However, as it is a prokaryotic channel, the VSD of KvLm contains only three of the eight conserved charged residues known to be deterministic for voltage sensing in eukaryotic $\mathrm{Kv}$ channels, which may impede the generalization of these findings 
to eukaryotic Kv channels $[15,16]$. To the best of our knowledge, similar protein engineering strategies (physically remove VSDs) have not been successful in eukaryotic Kv channels. The two-pore domain potassium (K2P) TASK3 channel is a dimeric protein, with each subunit consisting of four transmembrane segments flanked by a short N-terminal domain and a relatively longer C-terminal domain [17-22]. Different from $\mathrm{Kv}$ channels, dimeric TASK3 is categorized as open rectifier channel that does not exhibit activation, deactivation, and inactivation kinetics [23-25]. To investigate the roles of each single VSD, we developed a graft strategy to construct new channels using the TASK3 channel as the host and VSDs of voltage-gated ion channels as the donors. The VSDs dissected from tetrameric voltage-gated channels were grafted to the host TASK3 or its tandem channels, which produced functionally engineered channels with two or one VSDs, respectively. Using this graft strategy, we revealed distinctly new insight into the roles of VSDs of voltage-gated channels.

\section{Results}

Grafting VSDs of tetrameric voltage-gated channels to dimeric TASK3 channels

To produce voltage-gated channels with a reduced number of VSDs, a full-length dimeric TASK3 channel was used as the host. Each subunit of TASK3 channels contains two pore-forming domains but lacks a VSD (Figure 1A and Supplementary information, Figure S1). VSDs that contained a complete N-terminus, S1-S4 segments and a S4-S5 linker from three classical Kv channels (rKv1.2, hKv7.1, and rKv7.3) and a hyperpolarization-activated channel (mHCN1) were grafted to the host channel (Figure 1A and 1D, and Supplementary information, Figure S1). A total of four engineered channels, with each subunit containing one VSD, were constructed. Each engineered channel therefore theoretically consisted of two VSDs. These chimera channels were expressed in Chinese hamster ovary $(\mathrm{CHO})$ cells and examined using whole-cell electrophysiological recording (Figure 1B). Under voltage step stimulations, both significant outward and inward currents were elicited in the cells expressing mHCN1VSD-TASK3, rKv1.2VSD-TASK3 or rKv7.3VSD-TASK3 channels. However, under identical conditions, negligible inward currents were induced for the hKv7.1VSD-TASK3 (KT) channels. The rectification ratios $(\mathrm{R})$ of these engineered channels were calculated as the ratio of absolute current amplitude at $-100 \mathrm{mV}$ over that at $+100 \mathrm{mV}$. The rectification ratio of KT channels was significantly smaller than those of the other three channels (Figure 1C). The current-voltage (I-V) plots further revealed the apparent outward rectification of the KT channels (Supplementary information, Figure S2). Although the four donor VSDs exhibit topological similarity, including the conserved positively charged residues and some hydrophobic residues, the identity of their sequence was low (Figure 1D). Intriguingly, under the step pulses, all four engineered channels exhibited robust currents, suggesting a high host tolerance of TASK3 channels.

\section{The KT channels are voltage gated}

The KT channel was selected for further investigation because of its smallest rectification ratio and robust outward currents (Figure 1C). Under the same stimulation protocol, the elicited currents of TASK3 were distinct from those of Kv7.1 and KT channels (Figure 2A). Both large outward and inward currents were elicited by step stimulations, exhibiting a nearly linear $I-V$ relationship (Supplementary information, Figure S3A). The Kv7.1 channels, containing four VSDs, display a classical sigmoid $G-V$ curve. Interestingly, KT channels, containing grafted VSDs from Kv7.1, exhibited similar voltage dependence to Kv7.1 channels but dramatically different voltage dependence from TASK3 channels (Figure 2B). The $G-V$ curve of KT channels was also classically sigmoid, although the $V_{1 / 2}$ was right shifted to $+40.6 \pm 1.3$ $\mathrm{mV}$ from $-18.4 \pm 0.9 \mathrm{mV}(n=5, P<0.01)$ of $\mathrm{Kv} 7.1$ channels. The slope of $G-V$ curve of Kv7.1 channels was $\sim 17.0 \mathrm{mV}$. In contrast, the slope of $G-V$ curve of $\mathrm{KT}$ channels was shifted to $22.0 \mathrm{mV}$, indicating that the curve became shallower. The $G-V$ curve of TASK3 channels clearly showed that the conductance of the channel remains at a nearly constant level under stimulations ranging from $-100 \mathrm{mV}$ to $+50 \mathrm{mV}$. Although weak voltage dependence was observed at voltages more positive than $+50 \mathrm{mV}$, TASK 3 channels did not exhibit voltage-dependent closing (deactivation) under hyperpolarized membrane potentials. In contrast, the voltage-dependent deactivation was observed in both Kv7.1 and KT channels (Figure 2A and Supplementary information, Figure S3B and S3C). Taken together, these results indicate that KT channels are voltage gated.

\section{One KT channel consists of two VSDs}

Each dimeric TASK3 channel has two homogeneous subunits [24]. To determine the stoichiometry of KT channels, a single-molecule fluorescence photobleaching experiment using total internal reflection fluorescence (TIRF) microscopy was performed. The mEGFP tags were fused to the C-terminus of TASK 3 channels and the $\mathrm{N}$-terminus of the VSDs in KT channels, respectively (Figure 2C and 2D). To maintain the density of channels 

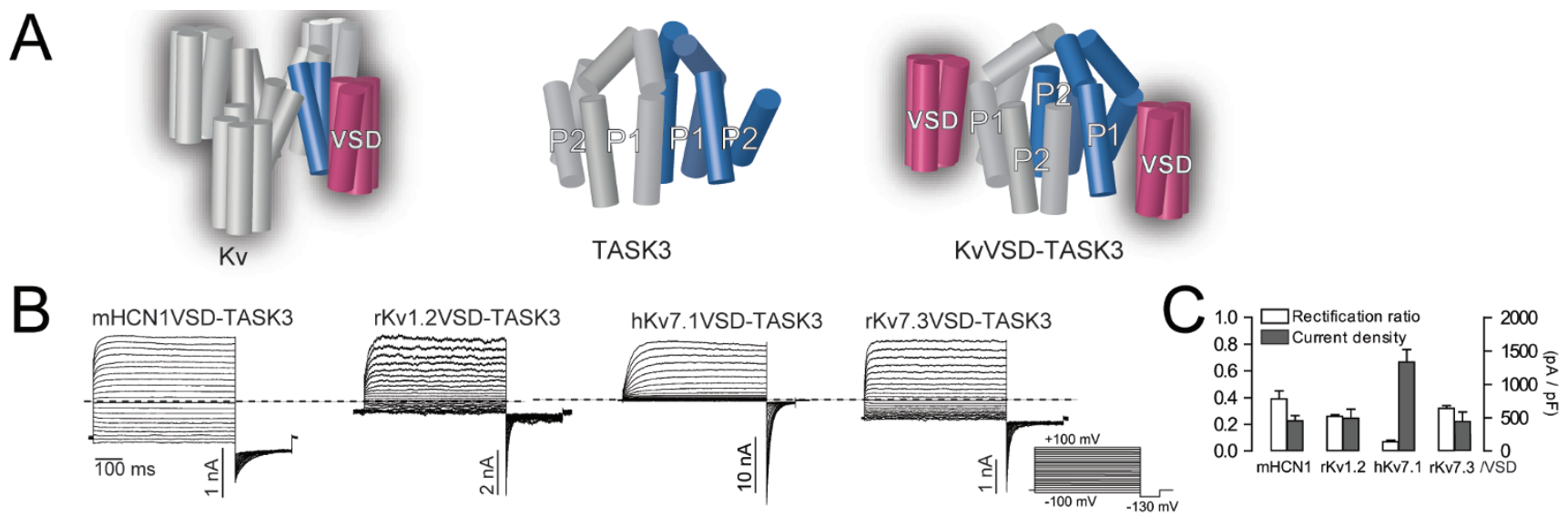

$\mathrm{D}$

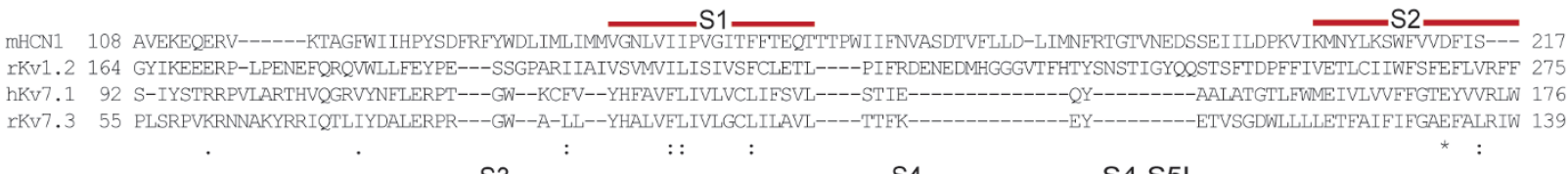

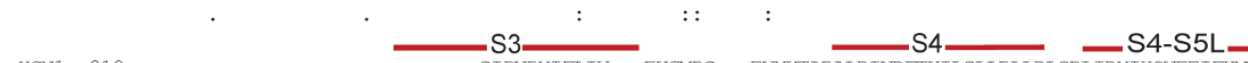

mHCN1 218 ---------------------------SIPVDYTFLTV---EKGMDS---EVYKTARALRIVRFTKILSLLRLLRLSRLIRYIHQWEEIFHMTYDLASAV-------------------- 284 rKV1.2 276 ACPSKA---GF-------FTNIMNIIDIVAIIPY-FITLGTELAEKPEDAQQGQQAMSLAILRVIRLVRVERIFKLSRHSKGLQILGQTLK--AS-------------------------------- 357 hKV7.1 177 SAGCRSKYVGLWGRLRFARKPISIIDLIVVVAS-MVVLCV---------GSK-GQVEATSAIRGIRFLQILRMLHVDRQGGTWRLLGSVVF--IH------------------------------- 258 rKV7.3 140 AAGCCCRYKGWRGRLKFARKPLCMLDIFVLIAS-VPVVAV--------GNQ-GNVL_AT-SLRSLRFLQILRMLRMDRRGGTWKLLGSAIC--AH----------------------------- 220

$$
:{ }^{*}: \quad: \text { : : : : }: \text { : }
$$

Figure 1 The GVTDT strategy. (A) Schematic representation of KvVSD-TASK3 channels. In Kv channels, four VSDs are coupled to a potassium-selective pore domain. In TASK3 channels, each subunit includes two pore-forming domains (P1 and P2) but lacks VSD. For clarity, only one subunit is colored. VSDs are colored red; pore domains are colored blue. The VSDs from different $\mathrm{Kv}$ channels were grafted to TASK3 channels by fusing the VSDs with the N-terminus of TASK3 channels. (B) Representative traces of the indicated KvVSD-TASK3 channels. The holding potential was $-90 \mathrm{mV}$. Whole-cell currents were elicited by $500-\mathrm{ms}$ pulses from -100 to $+100 \mathrm{mV}$ steps followed by a 200 -ms or 100 -ms hyperpolarization at $-130 \mathrm{mV}$ to record tail currents. The bath solution contained $140 \mathrm{mM} \mathrm{KCl}$ as described in Materials and Methods. (C) The rectification ratio and current density of the channels constructed with indicated KvVSD. See also Supplementary information, Figure S2. (D) Sequence alignment of VSDs of $\mathrm{mHCN} 1, \mathrm{rKv} 1.2, \mathrm{hKv7.1}$ and rKv7.3 channels. Same or similar residues were labeled with '*', ':' or ' ' SSee also Supplementary information, Figure S1.

low enough to minimize the chance of incidental overlap of two or more channels within a diffraction-limited area, the cells expressing TASK3 or KT channels were fixed only 3-5 $\mathrm{h}$ after transfection and examined by TIRF microcopy, as previously described [26, 27]. In the quiescent cells, the exogenously expressed channels exhibited a punctate appearance of fluorescence on the membrane surface. A defined continuous laser power was applied to induce stepwise photobleaching of the selected fluorescent spots $(20-200$ spots in a $10 \times 10 \mu \mathrm{m}$ TIRF illumination area). For each of the chosen fluorescent spots, the number of bleaching steps indicated the number of fluorescent mEGFP molecules in one channel. For TASK3 channels, $60 \%$ analyzed fluorescent spots (341 of 571) were bleached in two steps, which is consistent with the dimeric stoichiometry of TASK3 channels (Figure 2C). A similar bleaching pattern was observed for the KT channels. Approximately 59\% (290 of 489) spots were bleached in two steps, indicating that KT is a dimeric channel or that one KT channel consists of two VSDs (Figure 2D).

The voltage sensitivity of KT channels is conferred by the grafted VSDS

TASK3 channels show very weak voltage dependence when the potentials are more positive than $+50 \mathrm{mV}$, whereas KT channels with two grafted VSDs exhibit classical voltage sensitivity as Kv channels. Conformation transitions of the selectivity filter mediate the voltage-dependent inactivation of some Kv channels [28-32]. Whether the intrinsic properties of TASK3 selectivity are disrupted by the grafted VSDs leading to the voltage sensitivity of KT channels was investigated. The experimental reversal potentials of KT channels were measured (Supplementary information, Figure S4). Under different $\mathrm{K}^{+}$gradients $(5 \mathrm{mM}, 50 \mathrm{mM}$, and $140 \mathrm{mM})$, the experimental reversal potentials of KT channels were $-80.4 \pm$ $3.2,-19.1 \pm 0.9$ and $1.4 \pm 0.7 \mathrm{mV}$, respectively, which 


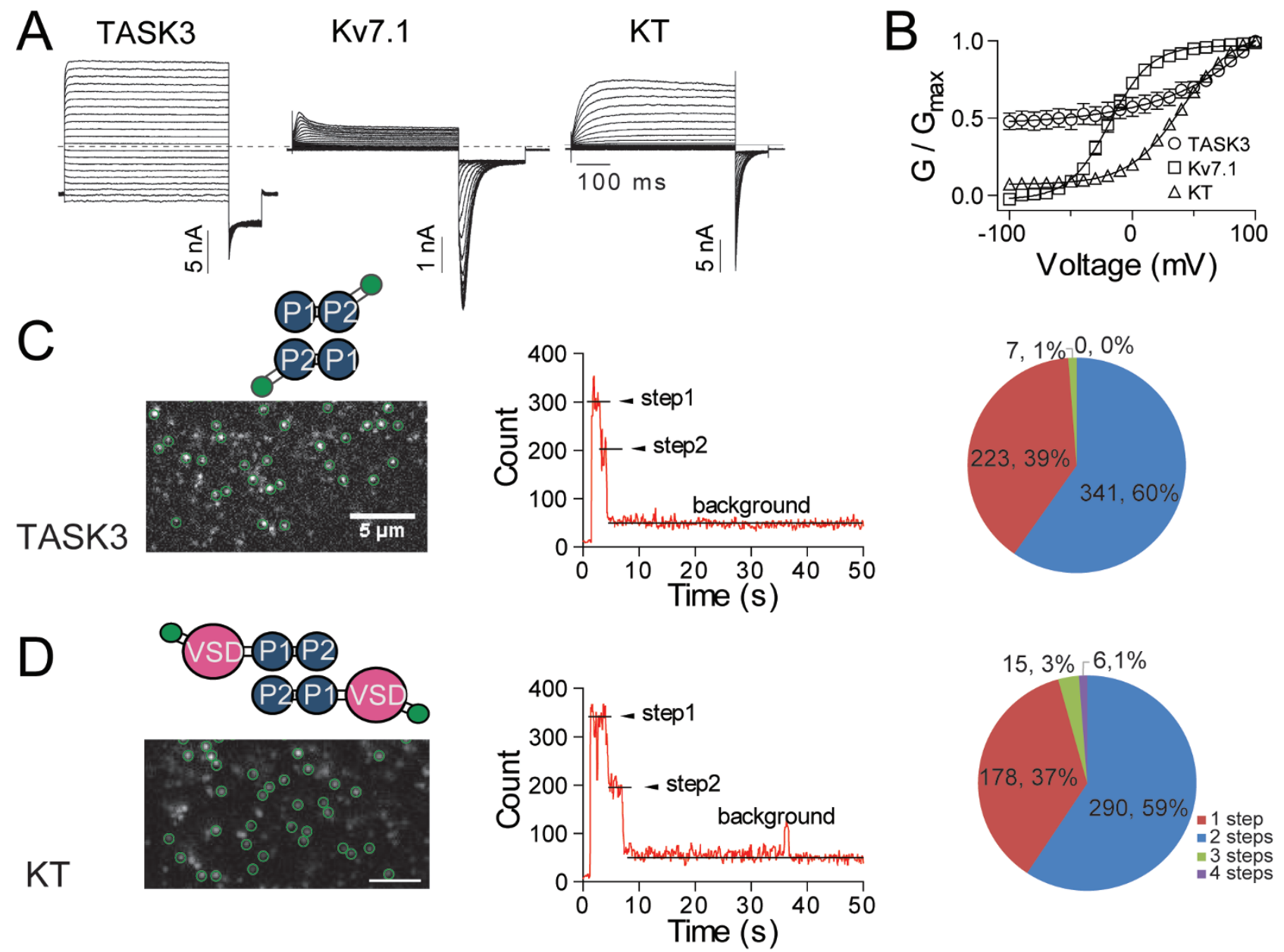

Figure 2 Characterization and stoichiometry of KT channels. (A) Whole-cell currents of TASK3 (left), Kv7.1 (middle), and KT (right) channels, which were recorded under identical conditions to those in Figure 1. (B) Normalized conductance-voltage (G-V) relationship of Kv7.1 (open square), TASK3 (open circle), and KT (open triangle). (C, D) Stoichiometry of TASK3 (C) and KT (D) channels. Left, representative images show TASK3 or KT puncta on the plasma membrane of a fixed cell. The puncta enclosed in green circles were chosen for single-molecule bleaching analysis. The pixel size of these images is 160 $\mathrm{nm}$, and the scale bar represents $1 \mu \mathrm{m}$. Schematic representations of TASK3-mEGFP and mEGFP-KT constructs are shown above the images. The green circles represent the tag mEGFP. Middle, representative time courses of mEGFP emission for the two constructs are shown after background correction. Right, distribution of bleaching steps of mEGFP-tagged TASK3 and KT channels. Majority of fluorescence spots on the plasma membrane were bleached in two steps.

were close to the theoretical $\mathrm{K}^{+}$equilibrium potentials, indicating that the $\mathrm{KT}$ channels are still $\mathrm{K}^{+}$-selective channels. TASK3 channels are intrinsically $\mathrm{pH}$ gated [23]. Similar to wild-type TASK3 channels, we found that KT channels were also sensitive to low $\mathrm{pH}$. The perfusion of acidic extracellular solutions $(\mathrm{pH}=5.5)$ completely inhibited the currents of both TASK 3 and KT channels (Figure 3). $\mathrm{H} 98$ is a $\mathrm{pH}$ sensor that resides near the extracellular mouth of the pore, and mutations of H98 inhibit the $\mathrm{pH}$ sensitivity of TASK 3 channels $[24,33]$. Notably, the mutation of $\mathrm{H} 98 \mathrm{~K}$ reduced the low-pH inhibition of both TASK3 and KT channels (Figure 3). These results indicate that the pore region of KT channels possesses similar intrinsic properties to that of TASK3 channels.

To investigate whether the voltage dependence of KT channels is derived from the grafted VSDs, we performed two independent experiments. The gating charge residues in $\mathrm{S} 4$ are the principal components to sense the voltage changes [34]. Neutralizing the first arginine (R228) of Kv7.1 S4 dramatically affects the voltage dependence of activation, whereas neutralizing the second arginine (R231) leads to loss of voltage dependence, characterized by a linear $G-V$ relationship [35-37]. The two corresponding positive residues in KT channels were mutated 

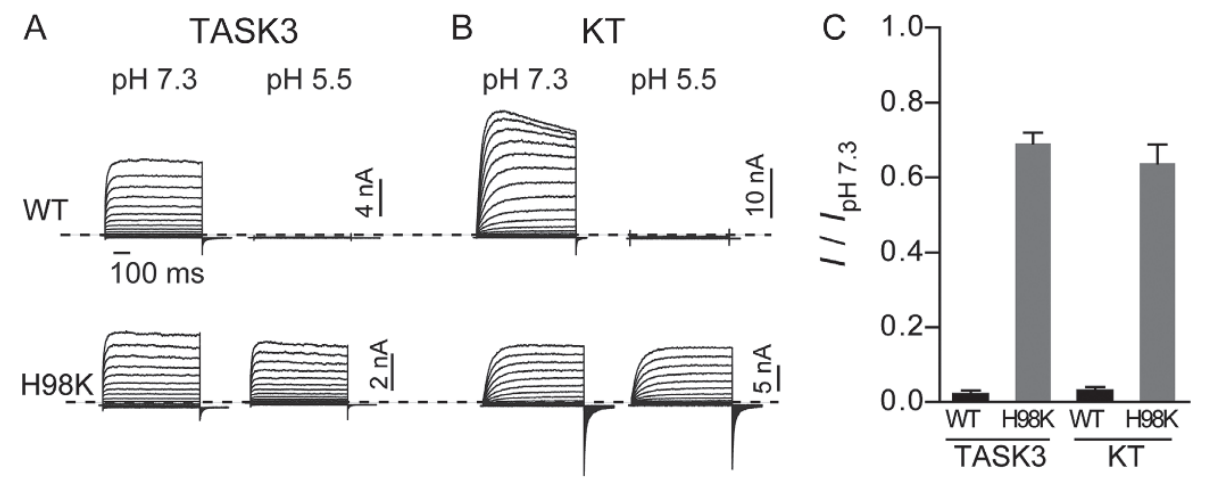

Figure $3 \mathrm{pH}$ sensitivity of TASK3 and KT channels. (A, B) Exemplary current traces recorded in CHO cells expressing wildtype (WT) TASK3, TASK3H98K, KT, or KTH98K channels in bath solution at pH 7.3 or 5.5. Currents were recorded in $5 \mathrm{mM}$ extracellular potassium (TASK3, TASK3H98K, and KT) or $140 \mathrm{mM}$ extracellular potassium (KTH98K) using 500-ms pulses from -100 to $+100 \mathrm{mV}$ steps from a holding potential of $-90 \mathrm{mV}$. (C) Histograms showing $I / I_{\mathrm{pH}} 7.3$ ratios of TASK3 (0.0 \pm 0.0$)$, TASK3H98K $(0.7 \pm 0.1)$, KT $(0.0 \pm 0.0)$, and KTH98K $(0.6 \pm 0.0)$ channels in $\mathrm{pH} 5.5$ and $\mathrm{pH} 7.3$ bath solution. The currents were measured at $0 \mathrm{mV}$.

to alanine (A). Interestingly, under identical conditions, the voltage dependence of the mutated KT channels was largely weakened in comparison with that of wildtype KT channels. Both the constitutive opening and the linear $G-V$ relationship were similar to those of TASK3 channels (Figure 4A and 4B). Benzbromarone (BBR) is a Kv7.1 activator that acts on VSDs [38]. The potentiation effects of BBR on Kv7.1 channels mainly included an increase of the outward currents and a left shift of the $G-V$ curve (Figure 4D, 4F and 4G). The compound BBR did not affect the TASK3 channels (Figure 4C and 4F). However, after grafting two VSDs, the KT channels became sensitive to BBR (Figure 4E, 4F, 4H and Supplementary information, Figure S5). Under treatment of $10 \mu \mathrm{M}$ BBR, the increases of the outward current $\left(I / I_{0}\right)$ at $0 \mathrm{mV}$ were $2.8 \pm 0.5$ versus $3.5 \pm 0.5$ for Kv7.1 and KT channels, respectively (Figure 4F). The left shift of the $G-V$ curves of Kv7.1 and KT channels by BBR treatment was also comparable (Figure 4G and $4 \mathrm{H}$ ). For Kv7.1 channels, the $V_{1 / 2}$ was shifted from $-1.9 \pm 2.0 \mathrm{mV}$ to $-22.1 \pm 2.9 \mathrm{mV}$ (Figure $4 \mathrm{G}$ ); for KT channels, the value was shifted from $76.0 \pm$ $4.5 \mathrm{mV}$ to $56.7 \pm 4.6 \mathrm{mV}$ (Figure $4 \mathrm{H}$ ). Taken together, these results indicate that the two grafted VSDs transfer voltage dependence and pharmacological sensitivity to KT channels.

One VSD is sufficient to confer voltage dependence and pharmacological sensitivity to Kv7.1-TASK3 channels

Voltage-gated KT channels consist of two grafted VSDs. We then attempted to produce a channel consisting of only one VSD. Tandem TASK3-TASK3 channels are functional [33]. A Kv7.1VSD-TASK3-TASK3 (KTT) channel was therefore constructed by grafting one Kv7.1
VSD to the tandem TASK3-TASK3 channel (Figure 5A and Supplementary information, Figure S1). For clarity, in the following text, the TASK3 proximal to the VSD was named T1; the distal TASK3 was named T2. To determine the number of VSDs in a KTT channel, single-molecule imaging was performed (Figure 5B). As in TASK3 and KT channels, the mEGFP tags were fused to the N-terminus of KTT VSDs. However, different from TASK3 and KT channels, most of the KTT fluorescent spots $(90 \%, 392$ of 435$)$ were bleached in one step, indicating that one KTT channel contains one VSD. We observed that $<10 \%$ of KTT fluorescent spots (39 of 435) were bleached in two steps. One reasonable interpretation is that some fluorescent spots may include two KTT channels due to diffraction-limited imaging resolution. The observation on N-terminally mEGFP-tagged tandem TASK3-TASK3 channels confirmed our speculation. Approximately $89 \%$ fluorescent spots of the tandem TASK3 channels were bleached in one step (229 of 260; Supplementary information, Figure S6). However, this two-step bleaching still raises a less likely possibility that the VSD and T1 may form a dimeric channel complex themselves in the same way as KT channels, with T2 flanking intracellularly. To exclude this possibility, the $\mathrm{pH}$ sensitivity of KTT channels consisting of one or two H98K mutations was then examined (Figure 5C). Briefly, the $\mathrm{pH}$-sensing residue $\mathrm{H} 98$ in $\mathrm{T} 2$ or in both $\mathrm{T} 1$ and $\mathrm{T} 2$ was mutated to lysine $(\mathrm{K})$. Then, the $\mathrm{pH}$ sensitivity of the two mutants, KTT(M) and $\mathrm{KT}(\mathrm{M}) \mathrm{T}(\mathrm{M})$, was evaluated and compared with that of wild-type KTT channels. The $\mathrm{pK}_{1 / 2}$ values of KTT and KTT(M) were $6.8 \pm 0.1$ and 5.9 \pm 0.0 , respectively, whereas the $\mathrm{pK}_{1 / 2}$ of $\mathrm{KT}(\mathrm{M}) \mathrm{T}(\mathrm{M})$ was lower than 5.0. Obviously, the H98K mutation in $\mathrm{T} 1$ 

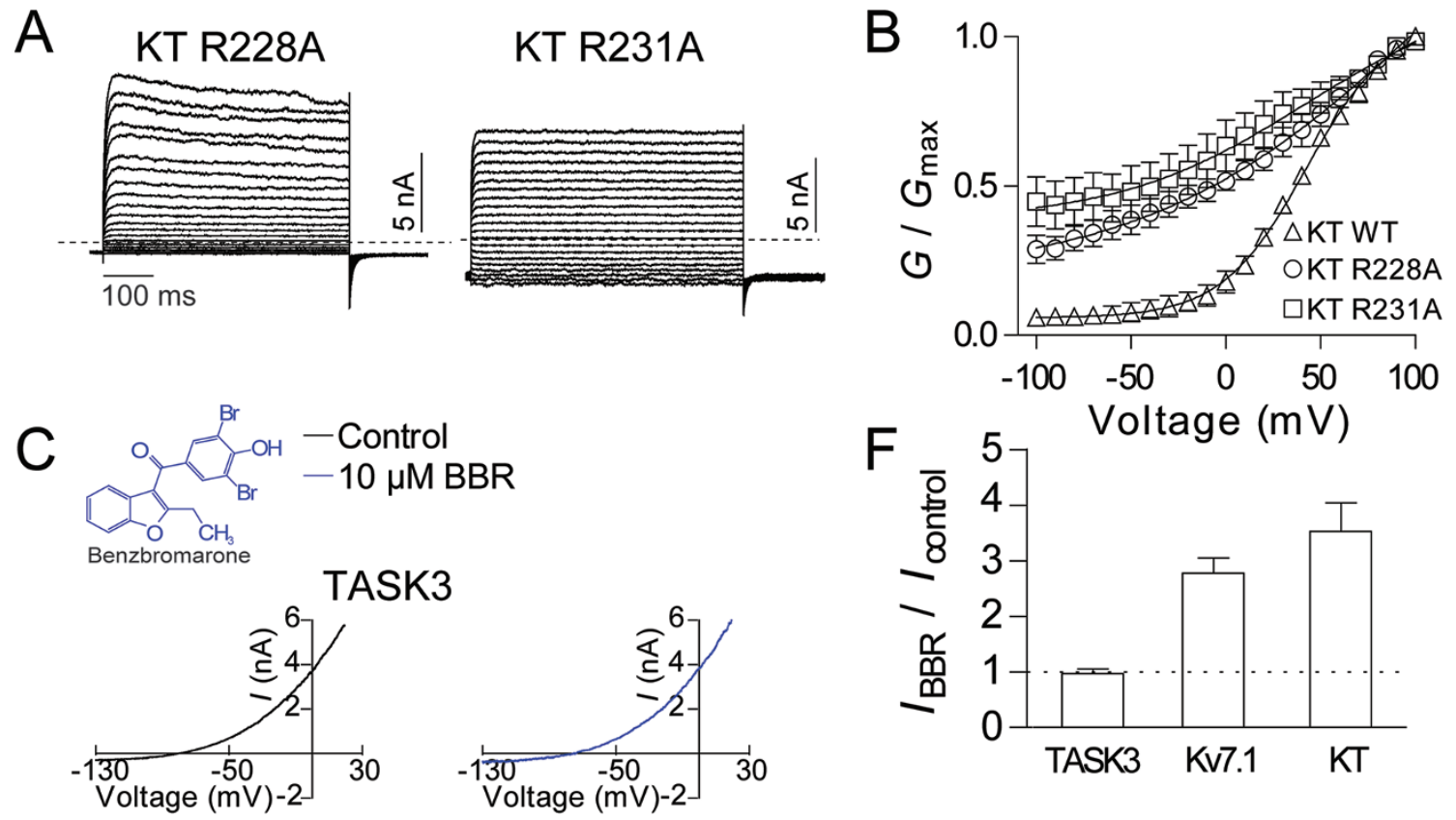

D
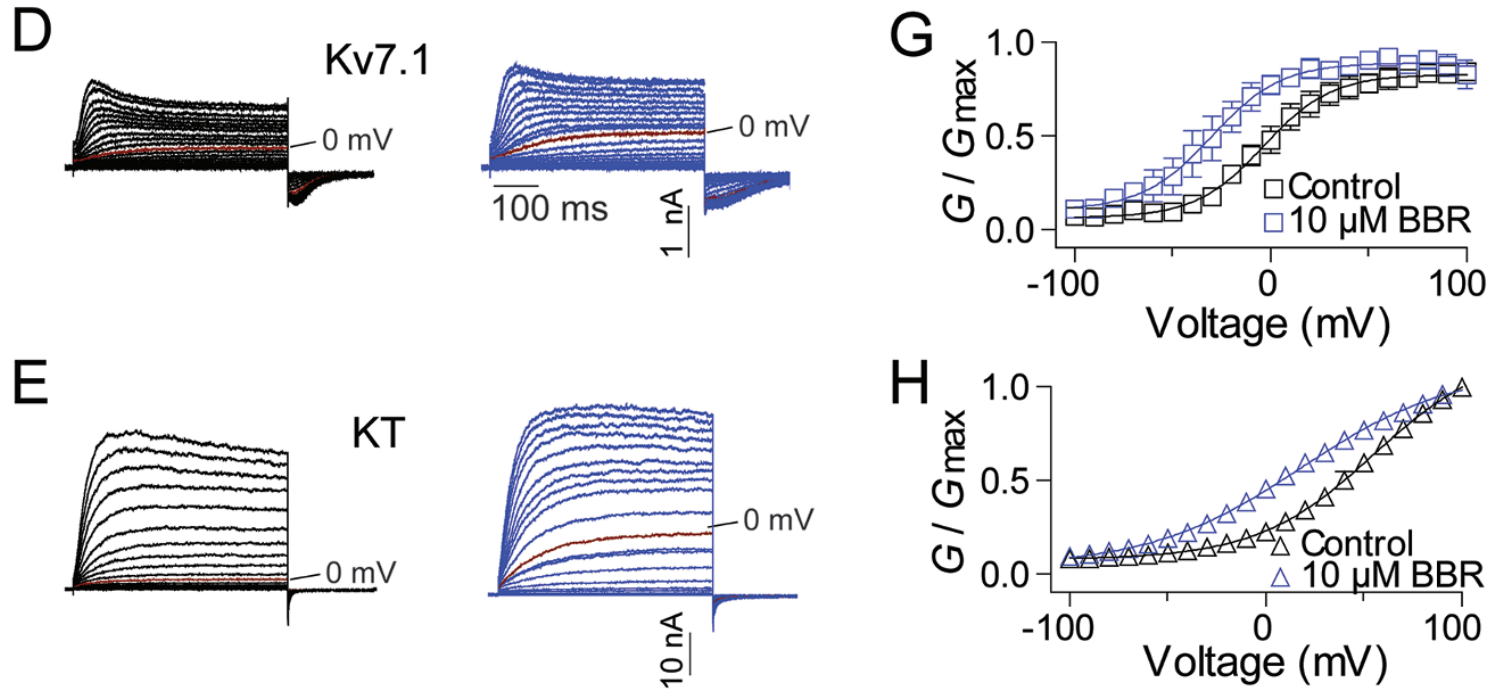

Figure 4 The grafted VSDs confer voltage and pharmacological sensitivity. (A) Representative traces of mutant KT R228A (left) and KT R231A (right) channels. (B) G-V curves of KT WT (open triangle), KT R228A (open circle) and KT R231A (open square) channels. (C-H) Effects of BBR on TASK3, Kv7.1 and KT channels. Currents of TASK3 channels (C) were elicited in the absence (black) and presence (blue) of $10 \mu \mathrm{M}$ BBR by $750-\mathrm{ms}$ ramp pulses from -130 to $+20 \mathrm{mV}$ from a holding potential of $-90 \mathrm{mV}$ with a 10-s interpulse interval. The chemical structure of BBR is shown. Currents of Kv7.1 (D) and KT (E) channels were elicited by $500-\mathrm{ms}$ pulses from -100 to $+100 \mathrm{mV}$ steps from a holding potential of $-90 \mathrm{mV}$ with a 2-s interpulse interval. Histogram for the current ratio $\left(I_{\mathrm{BBR}} / I_{\text {control }}\right)$ of TASK3 $(1.0 \pm 0.1, n=4), \mathrm{Kv} 7.1(2.8 \pm 0.3, n=7)$, and KT $(3.5 \pm 0.5, n=5)$ channels at $0 \mathrm{mV}(\mathbf{F})$. G-V curves of Kv7.1 (G) and KT (H) channels in the absence (black) and presence (blue) of $10 \mu \mathrm{M}$ BBR.

further decreased the $\mathrm{pH}$ sensitivity of the KT(M)T(M) channels, which is consistent with the previous report on tandem TASK3 channels [33]. Collectively, we successfully constructed a functional KTT channel consisting of one VSD.

The voltage dependence of KTT channels was eval- uated under identical conditions to that of KT channels. The currents of KTT channels are clearly voltage dependent, displaying slow activation and slow deactivation in the presence of either 5 or $140 \mathrm{mM}$ extracellular $\mathrm{K}^{+}$ (Figure 5D and 5E). Because of the limitation of cell tolerance and thus the lack of depolarization steps larger 
than $+100 \mathrm{mV}$, only a quasi-classical sigmoid $G-V$ curve was produced (Figure 5F). Compared with the $G-V$ curve of KT channels, the apparent $V_{1 / 2}$ of KTT channels was not right shifted, whereas the slope was changed from 22.0 to $37.0 \mathrm{mV}$, indicating the decreased voltage sensitivity of KTT channels. Then, whether a single VSD could transfer pharmacological sensitivity was examined. The $G-V$ curve of KTT channels was not changed after application of $10 \mu \mathrm{M}$ BBR (Supplementary information, Figure S5). However, at the same concentration, BBR increased the outward currents of KTT channels by $~ 3.5$ fold, which was comparable with its effect on Kv7.1 or KT channels (Figure 5G). Taken together, these results indicate that one single VSD is sufficient to confer the voltage and pharmacological sensitivities to voltage-dependent potassium channels.

\section{Discussion}

The GVTDT (Graft VSD To Dimeric TASK3) strategy was developed based on the modularity theory, which indicates that the pore and voltage sensors are distinct functional modules. In 2001, Lu et al. [39] demonstrated that the ion conduction pore is conserved among some potassium channels by substituting the prokaryotic pore into eukaryotic $\mathrm{Kv}$ channels. Later on, the voltage-sensing module S3b-S4 paddles of voltage-sensitive proteins were demonstrated to be transferable [40-44]. Interestingly, the analogous paddle of the non-voltage-gated cyclic nucleotide-gated ion (CNG) channels or TRP channels is also transplantable for $\mathrm{Kv}$ channels [45]. Most recently, fusion of the VSD of Ci-VSP to the viral channel $\mathrm{Kcv}$ created a functional voltage-gated $\mathrm{K}^{+}$channel [46].
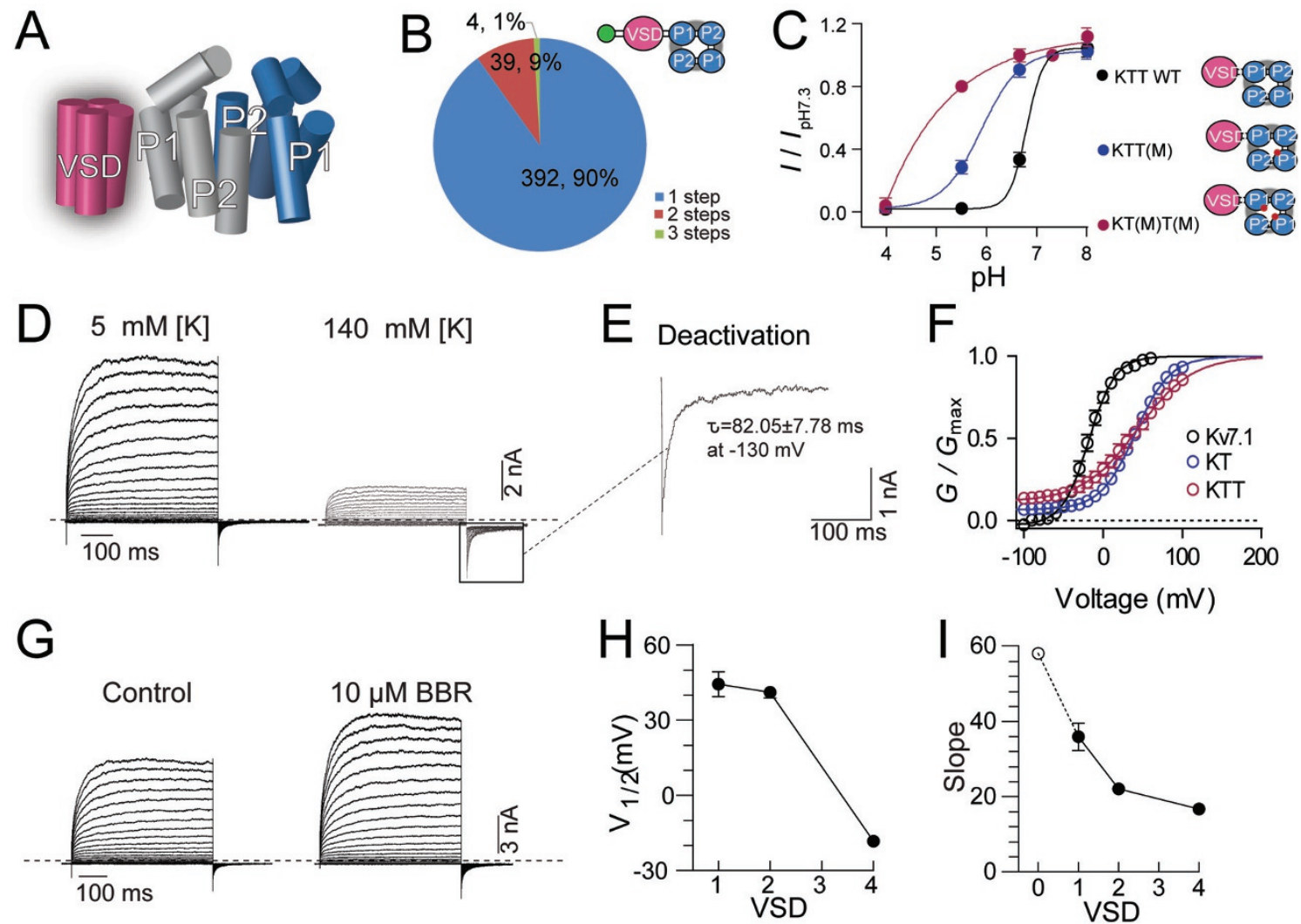

Figure 5 One VSD voltage-dependently gates a potassium channel. (A) Illustration depicting KTT channels. One Kv7.1VSD (red) was fused to a tandem TASK3 channel (blue) consisting of four pore domains (two P1 and two P2). (B) Stoichiometry of KTT channels. (C) pH sensitivity of KTT, KTT(M) and KT(M)T(M) channels. (D) Voltage sensitivity of KTT channels. Representative currents of KTT channels elicited in $5 \mathrm{mM}$ (left) and $140 \mathrm{mM}$ (right) extracellular potassium. (E) Representative tail currents recorded under $-130 \mathrm{mV}$ (black square indicated in D showing slow closing of KTT channels). (F) G-V curves of Kv7.1, KT, and KTT channels in $140 \mathrm{mM}$ extracellular potassium. (G) Representative currents of KTT channels in the absence (left) and presence (right) of $10 \mu \mathrm{M}$ BBR. Currents were elicited by 500 -ms pulses from -100 to $+100 \mathrm{mV}$ steps from a holding potential of $-90 \mathrm{mV}$. (H, I) Comparison of Kv7.1 (4 VSDs), KT (2 VSDs), KTT (1 VSD), and TASK3 (0 VSD) channels on $V_{1 / 2}(\mathbf{H})$, and the slope of $G-V$ curve (I) summarized from previous data. 
Notably, the S4-S5 linker is not required for voltage-dependent gating of $\mathrm{KCNH}$ potassium channels, suggesting that the voltage sensing could be transduced between the pore and VSD modules in the absence of covalent linker [47]. Although a single VSD can sense the changes of membrane potential and function independently [48-50], swapping the full-length VSD among 4-fold symmetric voltage-sensitive channels or transplanting the full-length VSD to the monomeric VSP failed to obtain functional proteins $[40,51]$. In our study, grafting the full-length VSDs, together with the upstream $\mathrm{N}$-terminus and the downstream S4-S5 linker, to the host protein as an integral module produced functional voltage-gated channels, extending our understanding of the modularity of VSD.

Some previous studies supported that the Kv pore is intrinsically more stable when closed, which distinguishes it from constitutively open K2P channels [52, 53]. Notably, the study on KvLm channels revealed that pore only module can populate at open state, suggesting that the pore of $\mathrm{Kv}$ channels may also reside in between closed-activated and open state [16]. The gating of the $\mathrm{Kv}$ channel pore involves both the lower bundle-crossing gate at the cytoplasmic entrance to the channel and the upper gate at the selectivity filter close to the outer mouth of the channel $[54,55]$. By contrast, the gating of K2P channels was thought to occur through their upper gate [56-59]. However, except for the upper gate, recent evidence shows that K2P channels may also possess a lower bundle-crossing gate, similar to Kv channels [59-61]. For TASK3 channels, the pore-lining helices consisting of two highly conserved putative hinge glycines form the lower gate and mutations of these glycines affect channel opening [62]. Therefore, the grafted VSDs may open or close the constructed KT or KTT channels by regulating the lower bundle-crossing gate of TASK3 channels.

Our study may reconcile some apparently conflicting observations on the gating of Kv channels. In Shaker family Kv channels, it is thought that the hyperpolarization of membrane potential would drive the VSDs down, causing the S4-S5 linkers to press on the outer side of the pore-lining helices, closing the lower gate $[63,64]$. By contrast, the VSDs are driven outward upon membrane depolarization, pulling on the S4-S5 linker and then opening the lower gate. There are four VSDs in a Kv channel, and it is therefore reasonable to deduce that they would work cooperatively during the gating process. Earlier experiments suggested that the transfer of the gating charges in all four VSDs must be sufficient to open the pore $[65,66]$. Immobilizing one $\mathrm{S} 4$ segment prevents Shaker activation gate from opening [67]. In line with these experimental measurements, a recent all-atom molecular simulation suggested that full outward move- ment of S4 in one or two VSDs is insufficient to open the pore [1]. The experiment in the prokaryotic KvLm channels further revealed that three VSDs are required to promote classical voltage-dependent channel opening at depolarizing potentials [16]. In our study, either one or two VSDs are sufficient to open the channels in a voltage-dependent manner. However, compared with the $G-V$ curve of the donor Kv7.1 channels with four VSDs, the KT (consisting of two VSDs) and KTT (consisting of one VSD) channels exhibit positively shifted $V_{1 / 2}$ and more shallow activation curve (Figure 5H and 5I), suggesting a reduction of voltage sensitivity. The voltage sensitivity of these three types of channels is positively correlated with the numbers of VSDs that they possess. Collectively, we propose that one VSD could be sufficient to voltage dependently open the channel, whereas the cooperation of VSDs facilitates the opening process and therefore increases the voltage sensitivity. Channel closure is an energetically asymmetric process relative to channel opening $[1,64]$. The off- and on-gating currents are usually different [68]. In particular, mutations in the S6 helices often affect the opening but not the closing kinetics $[69,70]$. In prokaryotic KvLm channels, four VSDs were required for full closure [16]. Interestingly, the studies in some $\mathrm{Kv}$ channels suggest that the intrinsically most stable state of the Kv pore is the closed state $[1,52,53]$. The VSDs thus do not need to actively push the S4-S5 linker down to close the pore because the pore cavity is highly hydrophobic [52]. Consistently, a molecular simulation further showed that the limited motion of a single VSD towards the resting state is sufficient to close the pore [1]. Our study incorporated these theoretical and experimental results. Both KT and KTT channels that contain either one or two VSDs clearly exhibit voltage-dependent deactivation in the same way as $\mathrm{Kv}$ channels.

In summary, the current study developed a strategy to construct voltage-gated ion channels with reduced number of VSDs. The finding that one VSD is sufficient to open and close the channels provides new insight into the roles of VSDs and refreshes our understanding of the gating of voltage-gated channels. Our work also provides a new method to gate TASK3, and perhaps other K2P channels. TASK3 shows a high host tolerance for VSDs from various $\mathrm{Kv}$ channels. KCNK2, another member of $\mathrm{K} 2 \mathrm{P}$ channels, could be converted to voltage-dependent channels by phosphorylation at a single canonical site for protein kinase A (PKA), which supports the idea that other K2P channels could also be receptive to the VSD chimeric strategy [71]. The novel channels constructed by fusing VSDs to different K2P channels would be essential for understanding the gating and regulation of the 
K2P channels.

\section{Materials and Methods}

\section{Plasmid construction}

The human Kv7.1 plasmid was a gift from Dr D Mackinnon (State University of New York, Stony Brook, USA). The human TASK3 was a gift from Dr Min Li (The Johns Hopkins University, USA). Point mutations were introduced using the QuikChange II site-directed mutagenesis kit (Stratagene) and the standard extension technique. The chimeric channels were generated by PCR using the overlap-extension method [72]. The cDNA encoding the chimeric channels was cloned into pMD18-T vectors (Takara) for amplification and sequencing. Then, all confirmed cDNAs were subcloned into pcDNA 3.1 for expression. TASK3 was tagged with mEGFP at the C-terminus through BamHI and Eco R I sites into pmEGFP-N1 vectors. KT and KTT were tagged at the N-terminus by subcloning mEGFP into a pcDNA3.1 backbone through NheI and BamHI.

\section{Cell culture and transient transfection}

CHO cells were grown in DMEM/F12 (Gibco) with 10\% FBS (Gibco). COS-7 cells were grown in DMEM with $10 \%$ FBS. Twenty-four hours before recording, cells were split and plated in 60-mm dishes, and were transfected with Lipofectamine 2000 reagent (Invitrogen) according to the manufacturer's instructions.

\section{Electrophysiological recording}

For current measurements of the channels expressed in $\mathrm{CHO}$ cells, standard whole-cell patch clamping was used at room temperature. The pipettes were pulled from borosilicate glass capillaries (World Precision Instruments). When filled with the intracellular solution, the pipettes had resistances of 3-5 M. During the recording, constant perfusion of extracellular solution was maintained using a BPS perfusion system (ALA Scientific). The pipette solution contained $140 \mathrm{mM} \mathrm{KCl}, 1 \mathrm{mM} \mathrm{MgCl}, 10 \mathrm{mM}$ EGTA, and $5 \mathrm{mM}$ HEPES; extracellular solution contained $135 \mathrm{mM} \mathrm{NaCl}$, $5 \mathrm{mM} \mathrm{KCl}$ (or $0 \mathrm{mM} \mathrm{NaCl}, 140 \mathrm{mM} \mathrm{KCl}$ or $90 \mathrm{mM} \mathrm{NaCl}$, and 50 $\mathrm{mM} \mathrm{KCl}$ ), $2 \mathrm{mM} \mathrm{CaCl}_{2}, 1 \mathrm{mM} \mathrm{MgCl}_{2}$, and $10 \mathrm{mM}$ HEPES [73]. The $\mathrm{pH}$ was adjusted to the desired values using $\mathrm{KOH}$ or $\mathrm{NaOH}$. Currents were recorded using an EPC-10 (HEKA) filtered at $2 \mathrm{kHz}$ and digitized using a DigiData 1440 with pClamp 9.2 software (Molecular Devices). Series resistance compensation and prediction were also used and set to $60 \%$. All chemicals were purchased from Sigma. Stock solutions $(20 \mathrm{mM})$ were prepared in dimethyl sulfoxide and diluted in the extracellular solution before use. Normalized conductance-voltage $(G-V)$ relationships were fitted using the Boltzmann equation. The slope describes the steepness of the curve, with a larger value denoting a shallow curve. Data analysis was performed using CLAMPFIT 9 (Axon Instruments) and GraphPad Prism 5.

\section{Single-molecule imaging}

The imaging experiments were performed using fixed COS-7 cells. A TIRF microscope, equipped with a high-NA TIRF objective (Olympus Oil $100 \times$ or $150 \times \mathrm{NA}=1.45$ ) and electron-multiplying charge-coupled device (AndoriXon DV-897 BV), was adopted to achieve single-molecule detection. mEGFP was excited with a solid-state 488-nm laser (OPSL, Coherent) with 1-mW laser power at the back pupil of the objective. Image stacks of 1 000 frames were acquired at $20 \mathrm{~Hz}$. Imaging data were analyzed by a home-written MATLAB code. Briefly, an àtrous wavelet filter was applied to each frame to extract all the single molecules. Each isolated molecules were fitted to a 2D Gaussian function to obtain the precise $x-y$ location, as well as the intensity and background. To find the corresponding single molecule in successive frames, a well-established trajectory linking algorithm (http://site.physics. georgetown.edu/matlab/) was adopted. For each identified trace, the bleaching steps were determined manually by one investigator and rescored blindly by another. Other criteria were as described in [26].

\section{Statistics}

The data are presented as means \pm SEM and the significance was estimated using paired or unpaired two-tailed Student's $t$-tests unless otherwise stated. Statistical significance: ${ }^{*} P<0.05, * * P<$ $0.01, * * * P<0.001$.

\section{Acknowledgments}

We gratefully acknowledge the financial support from the State Key Program of Basic Research of China (2013CB910604), the National Science and Technology Major Project on 'Key New Drug Creation and Manufacturing Program' (2013ZX09103001016), the National Natural Science Foundation of China grant for Excellent Key Laboratory (81123004), the National Natural Science Foundation of China (61327014, 91413122, 61175103, 31127901, 31127002 and 81461148027), Shanghai Municipal Science and Technology Commission (13JC1406700), and the External Cooperation Program of BIC, Chinese Academy of Sciences (1536631KYSB20130003).

\section{Author Contributions}

$\mathrm{XL}$ and FT performed electrophysiological and molecular biological experiments. CF, XL and WJ contributed to imaging of single molecules. ZG, TX and XL designed the experiments, and wrote the manuscript.

\section{Competing Financial Interests}

The authors declare no competing financial interests.

\section{References}

1 Jensen MO, Jogini V, Borhani DW, Leffler AE, Dror RO, Shaw DE. Mechanism of voltage gating in potassium channels. Science 2012; 336:229-233.

2 Papazian DM, Timpe LC, Jan YN, Jan LY. Alteration of voltage-dependence of Shaker potassium channel by mutations in the S4 sequence. Nature 1991; 349:305-310.

3 Aggarwal SK, MacKinnon R. Contribution of the S4 segment to gating charge in the Shaker $\mathrm{K}^{+}$channel. Neuron 1996; 16:1169-1177.

4 Liman ER, Hess P, Weaver F, Koren G. Voltage-sensing residues in the $\mathrm{S} 4$ region of a mammalian $\mathrm{K}^{+}$channel. Nature 1991; 353:752-756.

5 Lopez GA, Jan YN, Jan LY. Hydrophobic substitution mutations in the $\mathrm{S} 4$ sequence alter voltage-dependent gating in Shaker $\mathrm{K}^{+}$channels. Neuron 1991; 7:327-336. 
6 Seoh SA, Sigg D, Papazian DM, Bezanilla F. Voltage-sensing residues in the S2 and S4 segments of the Shaker $\mathrm{K}^{+}$channel. Neuron 1996; 16:1159-1167.

7 Long SB, Campbell EB, MacKinnon R. Voltage sensor of kv1.2: structural basis of electromechanical coupling. Science 2005; 309:903-908.

8 Ramsey IS, Moran MM, Chong JA, Clapham DE. A voltage-gated proton-selective channel lacking the pore domain. Nature 2006; 440:1213-1216.

9 Sasaki M, Takagi M, Okamura Y. A voltage sensor-domain protein is a voltage-gated proton channel. Science 2006; 312:589-592.

10 Murata Y, Iwasaki H, Sasaki M, Inaba K, Okamura Y. Phosphoinositide phosphatase activity coupled to an intrinsic voltage sensor. Nature 2005; 435:1239-1243.

11 Swartz KJ. Sensing voltage across lipid membranes. Nature 2008; 456:891-897.

12 Hodgkin AL, Huxley AF. A quantitative description of membrane current and its application to conduction and excitation in nerve. J Physiol 1952; 117:500-544.

13 Gagnon DG, Bezanilla F. A single charged voltage sensor is capable of gating the Shaker $\mathrm{K}^{+}$channel. J Gen Physiol 2009; 133:467-483.

14 Horn R. Uncooperative voltage sensors. J Gen Physiol 2009; 133:463-466.

15 Santos JS, Lundby A, Zazueta C, Montal M. Molecular template for a voltage sensor in a novel $\mathrm{K}+$ channel. I. Identification and functional characterization of KvLm, a voltage-gated $\mathrm{K}+$ channel from Listeria monocytogenes. J Gen Physiol 2006; 128:283-292.

16 Syeda R, Santos JS, Montal M, Bayley H. Tetrameric assembly of KvLm K+ channels with defined numbers of voltage sensors. Proc Natl Acad Sci USA 2012; 109:16917-16922.

17 Goldstein SA, Bockenhauer D, O'Kelly I, Zilberberg N. Potassium leak channels and the KCNK family of two-P-domain subunits. Nat Rev Neurosci 2001; 2:175-184.

18 Lesage F, Lazdunski M. Molecular and functional properties of two-pore-domain potassium channels. Am J Physiol Renal Physiol 2000; 279:F793-F801.

19 Kollewe A, Lau AY, Sullivan A, Roux B, Goldstein SA. A structural model for K2P potassium channels based on 23 pairs of interacting sites and continuum electrostatics. $J$ Gen Physiol 2009; 134:53-68.

20 Brohawn SG, del Marmol J, MacKinnon R. Crystal structure of the human K2P TRAAK, a lipid- and mechano-sensitive K+ ion channel. Science 2012; 335:436-441.

21 Dong YY, Pike AC, Mackenzie A, et al. K2P channel gating mechanisms revealed by structures of TREK-2 and a complex with Prozac. Science 2015; 347:1256-1259.

22 Miller AN, Long SB. Crystal structure of the human two-pore domain potassium channel K2P1. Science 2012; 335:432-436.

23 Duprat F, Lesage F, Fink M, Reyes R, Heurteaux C, Lazdunski M. TASK, a human background $\mathrm{K}+$ channel to sense external $\mathrm{pH}$ variations near physiological pH. EMBO J 1997; 16:5464-5471.

24 Rajan S, Wischmeyer E, Xin Liu G, et al. TASK-3, a novel tandem pore domain acid-sensitive $\mathrm{K}+$ channel. An extracellular histiding as pH sensor. $J$ Biol Chem 2000; 275:1665016657.
25 Leonoudakis D, Gray AT, Winegar BD, et al. An open rectifier potassium channel with two pore domains in tandem cloned from rat cerebellum. J Neurosci 1998; 18:868-877.

$26 \mathrm{Ji} \mathrm{W}, \mathrm{Xu} \mathrm{P}, \mathrm{Li} \mathrm{Z}$, et al. Functional stoichiometry of the unitary calcium-release-activated calcium channel. Proc Natl Acad Sci USA 2008; 105:13668-13673.

27 Ulbrich MH, Isacoff EY. Subunit counting in membrane-bound proteins. Nat Methods 2007; 4:319-321.

28 Cuello LG, Jogini V, Cortes DM, Perozo E. Structural mechanism of C-type inactivation in $\mathrm{K}(+)$ channels. Nature 2010; 466:203-208.

29 Cuello LG, Jogini V, Cortes DM, et al. Structural basis for the coupling between activation and inactivation gates in $\mathrm{K}(+)$ channels. Nature 2010; 466:272-275.

30 Labro AJ, Lacroix JJ, Villalba-Galea CA, Snyders DJ, Bezanilla F. Molecular mechanism for depolarization-induced modulation of Kv channel closure. J Gen Physiol 2012; 140:481-493.

31 Panaghie G, Purtell K, Tai KK, Abbott GW. Voltage-dependent C-type inactivation in a constitutively open $\mathrm{K}+$ channel. Biophys J 2008; 95:2759-2778.

32 Kiss L, LoTurco J, Korn SJ. Contribution of the selectivity filter to inactivation in potassium channels. Biophys $J 1999$; 76:253-263.

33 Gonzalez W, Zuniga L, Cid LP, Arevalo B, Niemeyer MI, Sepulveda FV. An extracellular ion pathway plays a central role in the cooperative gating of a $\mathrm{K}(2 \mathrm{P}) \mathrm{K}+$ channel by extracellular pH. J Biol Chem 2013; 288:5984-5991.

34 Chanda B, Asamoah OK, Blunck R, Roux B, Bezanilla F. Gating charge displacement in voltage-gated ion channels involves limited transmembrane movement. Nature 2005; 436:852-856.

35 Panaghie G, Abbott GW. The role of S4 charges in voltage-dependent and voltage-independent KCNQ1 potassium channel complexes. J Gen Physiol 2007; 129:121-133.

36 Osteen JD, Barro-Soria R, Robey S, Sampson KJ, Kass RS, Larsson HP. Allosteric gating mechanism underlies the flexible gating of KCNQ1 potassium channels. Proc Natl Acad Sci USA 2012; 109:7103-7108.

37 Liin SI, Barro-Soria R, Larsson HP. The KCNQ1 channel - remarkable flexibility in gating allows for functional versatility. J Physiol 2015; 593:2605-2615.

38 Zheng Y, Xu H, Zhan L, Zhou X, Chen X, Gao Z. Activation of peripheral KCNQ channels relieves gout pain. Pain 2015; 156:1025-1035.

$39 \mathrm{Lu}$ Z, Klem AM, Ramu Y. Ion conduction pore is conserved among potassium channels. Nature 2001; 413:809-813.

40 Alabi AA, Bahamonde MI, Jung HJ, Kim JI, Swartz KJ. Portability of paddle motif function and pharmacology in voltage sensors. Nature 2007; 450:370-375.

41 Jiang Y, Lee A, Chen J, et al. X-ray structure of a voltage-dependent K+ channel. Nature 2003; 423:33-41.

42 Jiang Y, Ruta V, Chen J, Lee A, MacKinnon R. The principle of gating charge movement in a voltage-dependent $\mathrm{K}+$ channel. Nature 2003; 423:42-48.

43 Bosmans F, Martin-Eauclaire MF, Swartz KJ. Deconstructing voltage sensor function and pharmacology in sodium channels. Nature 2008; 456:202-208.

44 Lee SY, Lee A, Chen J, MacKinnon R. Structure of the KvAP 
voltage-dependent $\mathrm{K}+$ channel and its dependence on the lipid membrane. Proc Natl Acad Sci USA 2005; 102:15441-15446.

45 Kalia J, Swartz KJ. Exploring structure-function relationships between TRP and Kv channels. Sci Rep 2013; 3:1523.

46 Arrigoni C, Schroeder I, Romani G, Van Etten JL, Thiel G, Moroni A. The voltage-sensing domain of a phosphatase gates the pore of a potassium channel. J Gen Physiol 2013; 141:389-395.

47 Lorinczi E, Gomez-Posada JC, de la Pena P, et al. Voltage-dependent gating of $\mathrm{KCNH}$ potassium channels lacking a covalent link between voltage-sensing and pore domains. Nat Commun 2015; 6:6672.

48 Kohout SC, Ulbrich MH, Bell SC, Isacoff EY. Subunit organization and functional transitions in Ci-VSP. Nat Struct Mol Biol 2008; 15:106-108.

49 Lee SY, Letts JA, Mackinnon R. Dimeric subunit stoichiometry of the human voltage-dependent proton channel Hv1. Proc Natl Acad Sci USA 2008; 105:7692-7695.

50 Tombola F, Ulbrich MH, Isacoff EY. The voltage-gated proton channel Hv1 has two pores, each controlled by one voltage sensor. Neuron 2008; 58:546-556.

51 Mishina Y, Mutoh H, Knopfel T. Transfer of Kv3.1 voltage sensor features to the isolated Ci-VSP voltage-sensing domain. Biophys J 2012; 103:669-676.

52 Jensen MO, Borhani DW, Lindorff-Larsen K, et al. Principles of conduction and hydrophobic gating in $\mathrm{K}+$ channels. Proc Natl Acad Sci USA 2010; 107:5833-5838.

53 Yifrach O, MacKinnon R. Energetics of pore opening in a voltage-gated $\mathrm{K}(+)$ channel. Cell 2002; 111:231-239.

54 del Camino D, Holmgren M, Liu Y, Yellen G. Blocker protection in the pore of a voltage-gated $\mathrm{K}+$ channel and its structural implications. Nature 2000; 403:321-325.

55 Liu Y, Holmgren M, Jurman ME, Yellen G. Gated access to the pore of a voltage-dependent K+ channel. Neuron 1997; 19:175-184.

56 Niemeyer MI, Gonzalez-Nilo FD, Zuniga L, Gonzalez W, Cid LP, Sepulveda FV. Neutralization of a single arginine residue gates open a two-pore domain, alkali-activated $\mathrm{K}+$ channel. Proc Natl Acad Sci USA 2007; 104:666-671.

57 Zilberberg N, Ilan N, Goldstein SA. KCNKO: opening and closing the 2-P-domain potassium leak channel entails "C-type" gating of the outer pore. Neuron 2001; 32:635-648.

58 Cohen A, Ben-Abu Y, Hen S, Zilberberg N. A novel mechanism for human K2P2.1 channel gating. Facilitation of C-type gating by protonation of extracellular histidine residues. $J$ Biol Chem 2008; 283:19448-19455.

59 Ben-Abu Y, Zhou Y, Zilberberg N, Yifrach O. Inverse cou- pling in leak and voltage-activated $\mathrm{K}+$ channel gates underlies distinct roles in electrical signaling. Nat Struct Mol Biol 2009; 16:71-79.

60 Mathie A, Al-Moubarak E, Veale EL. Gating of two pore domain potassium channels. J Physiol 2010; 588:3149-3156.

61 Piechotta PL, Rapedius M, Stansfeld PJ, et al. The pore structure and gating mechanism of K2P channels. EMBO J 2011; 30:3607-3619.

62 Ashmole I, Vavoulis DV, Stansfeld PJ, et al. The response of the tandem pore potassium channel TASK-3 (K(2P)9.1) to voltage: gating at the cytoplasmic mouth. J Physiol 2009; 587:4769-4783.

63 Long SB, Campbell EB, Mackinnon R. Voltage sensor of Kv1.2: structural basis of electromechanical coupling. Science 2005; 309:903-908.

64 Fowler PW, Sansom MS. The pore of voltage-gated potassium ion channels is strained when closed. Nat Commun 2013; 4:1872.

65 Schoppa NE, McCormack K, Tanouye MA, Sigworth FJ. The size of gating charge in wild-type and mutant Shaker potassium channels. Science 1992; 255:1712-1715.

66 Islas LD, Sigworth FJ. Voltage sensitivity and gating charge in Shaker and Shab family potassium channels. J Gen Physiol 1999; 114:723-742.

67 Horn R, Ding S, Gruber HJ. Immobilizing the moving parts of voltage-gated ion channels. J Gen Physiol 2000; 116:461476.

68 Batulan Z, Haddad GA, Blunck R. An intersubunit interaction between S4-S5 linker and S6 is responsible for the slow off-gating component in Shaker $\mathrm{K}^{+}$channels. J Biol Chem 2010; 285:14005-14019.

69 Stefani E, Toro L, Perozo E, Bezanilla F. Gating of Shaker $\mathrm{K}^{+}$channels: I. Ionic and gating currents. Biophys $J$ 1994; 66:996-1010.

70 Zagotta WN, Hoshi T, Aldrich RW. Shaker potassium channel gating. III: Evaluation of kinetic models for activation. J Gen Physiol 1994; 103:321-362.

71 Bockenhauer D, Zilberberg N, Goldstein SA. KCNK2: reversible conversion of a hippocampal potassium leak into a voltage-dependent channel. Nat Neurosci 2001; 4:486-491.

72 Heckman KL, Pease LR. Gene splicing and mutagenesis by PCR-driven overlap extension. Nat Protoc 2007; 2:924-932.

73 Ma L, Zhang X, Chen H. TWIK-1 two-pore domain potassium channels change ion selectivity and conduct inward leak sodium currents in hypokalemia. Sci Signal 2011; 4:ra37.

(Supplementary information is linked to the online version of the paper on the Cell Research website.) 